\title{
Nano-3D-printed Photochromic Objects
}

Sebastian Ulrich ${ }^{1,3}$, Xiaopu Wang ${ }^{2,6}$, Markus Rottmar ${ }^{4}$, René Michel Rossi ${ }^{1}$, Bradley J. Nelson ${ }^{2}$, Nico Bruns ${ }^{3}$, Ralph Müller ${ }^{5}$, Katharina Maniura-Weber ${ }^{4}$, Xiao-Hua Qin ${ }^{4,5} *$ and Luciano Fernandes Boesel ${ }^{1}$

${ }^{1}$ Empa, Swiss Federal Laboratories for Materials Science and Technology, Laboratory for Biomimetic Membranes and Textiles

Lerchenfeldstrasse 5, 9014 St. Gallen, Switzerland

${ }^{2}$ Institute of Robotics and Intelligent Systems

ETH Zurich

Tannenstrasse 3, 8092 Zurich, Switzerland

${ }^{3}$ Adolphe Merkle Institute

University of Fribourg

Chemin des Verdiers 4, 1700 Fribourg, Switzerland

${ }^{4}$ Empa, Swiss Federal Laboratories for Materials Science and Technology

Biointerfaces

Lerchenfeldstrasse 5, 9014 St. Gallen, Switzerland

${ }^{5}$ Institute for Biomechanics

ETH Zurich

Leopold-Ruzicka-Weg 4, 8093 Zurich, Switzerland

${ }^{6}$ Shenzhen Institute of Artificial Intelligence and Robotics for Society

The Chinese University of Hong Kong, Shenzhen

518172 Shenzhen, China

E-mail: qinx@ethz.ch

Keywords: Two-photon polymerization, nanoscale 3D-printing, thiol-ene reaction, donoracceptor stenhouse adducts, photochromism 


\begin{abstract}
A new class of photoresist is described for direct laser writing of photoswitchable 3D microstructures. The material comprising off-stoichiometric thiol-ene photo-clickable resins enables rapid two-photon laser processing of highly complex structures and facile postmodification with photoswitches. The microstructures were functionalized with a series of donor-acceptor Stenhouse adducts (DASAs) photoswitches with different excitation wavelength. The versatility of thiol-ene photo-click reaction enabled fine-tuning of the network structure and physical properties as well as the type and concentration of DASA photoswitches. When exposed to visible light, these microstructures exhibit excellent photo-responsiveness and undergo reversible color-changing via photoisomerization of DASA moieties. We describe that the weak fluorescence of DASAs can be used as a reporter of photoswitching, color changes, and thermal recovery, allowing the reading of DASA-containing sub-micrometric structures in 3D. This work delivers a new approach for custom microfabrication of 3D photochromic objects with molecularly engineered color and responsiveness.
\end{abstract}




\section{Introduction}

Stimuli-responsive materials based on organic photoswitches can reversibly change color and other properties when a light stimulus is applied, enabling their remote control with high spatial and temporal precision.[1-3] The controlled change of properties upon light irradiation has been exploited for a broad range of applications ranging from photochromic ophthalmic lenses to optical data storage, optical switches, phase shifters, sensors, drug delivery and actuators for soft robotics.[4-8] Most established systems, however, require UV light, which provides a very limited penetration depth into many materials, including skin, and is often detrimental to their structure.[9] These limitations have boosted the search for visible lightresponsive photoswitches. One the most remarkable developments in this field was the discovery of donor-acceptor Stenhouse adducts (DASAs), a new class of visible lightresponsive photoswitches with negative photochromism.[10-12] With a rapidly increasing knowledge on their structure-property relationships and their subsequent optimization, $2^{\text {nd }}$ and $3^{\text {rd }}$ generations of DASAs have overcome initial limitations, providing now access to the whole range of the visible spectrum with excellent photoswitching properties both in solution and in polymer matrices.[13-15] In materials science, DASA have already been employed in multiple applications from sensors to wavelength-specific photopatterning and visible light responsive enzyme nanoreactors.[16-26] In such multi-addressable materials, DASAs serve as molecular logic gates that only require different visible light sources to control the different states.[27, 28] To date, however, DASAs have been studied mostly in two-dimensional (2D) photoswitchable substrates such as polymer films and modified surfaces. Combining the wavelength-specific activation of DASA with complex-shaped objects could allow the fabrication of materials with sophisticated control both on the functional and the structural levels. Yet, approaches to fabricate customized 3D photochromic objects based on DASAs have to be established first.

Recent advances in 3D-printing technologies have witnessed their burgeoning applications in digital fabrication, microelectronics and biomedicine.[29-31] One of the most precise 3D- 
printing technologies is two-photon photolithography $(2 \mathrm{PP}),[32,33]$ which relies on localized curing of liquid ink materials upon non-linear two-photon photoinitiation triggered by an ultrafast near-infrared laser beam. Since most polymeric materials are transparent at $800 \mathrm{~nm}$, one can easily build custom 3D micro-objects in accordance with computer-aided design,[32] or even control material properties in space and time at submicron-level resolution.[33, 34] Radical-mediated thiol-ene click reactions are particularly suitable for photocrosslinking due to their high reactivity and selectivity through the fast initiation with radical photoinitiators and subsequent chain-transfer reactions.[35, 36] While 2PP has been used to fabricate optical devices such as waveguides,[37] so far there are no reports on 3D-printed photochromic microobjects that allow localized dynamic tuning of color on demand by visible light.

Herein, we describe a novel approach to create visible light-responsive photochromic 3D micro-objects by interfacing ink formulations containing DASAs with nanoscale 3D-printing technique based on 2PP. We firstly designed and formulated a highly efficient thiol-ene photoclickable ink system that consists of multifunctional thiols/enes and an ene-terminated DASA precursor. This ink system is modular and its robustness for thiol-ene photocrosslinking enables ultrafast laser microfabrication of complex $3 \mathrm{D}$ objects at high writing speeds up to $50 \mathrm{~mm} \cdot \mathrm{s}^{-1}$. The microstructures can be functionalized with a series of DASA photoswitches with wavelength-specific switching properties in a modular manner. By scanning a focused green laser inside the functionalized microstructure, we demonstrate photodynamic color-tuning of DASA-containing polymer networks at high spatial resolution as well as their reversible photoswitching. We demonstrate that the weak fluorescence of DASAs can be used as a reporter of photoswitching and color changes in sub-micrometric structures, for which standard techniques (e.g., UV-Vis spectrometry) are difficult to apply, either because the structures are too small or because they require 3D-reading capability.

\section{Results}


We designed a modular ink system to print visible light-responsive photochromic DASAnetworks by radical thiol-ene photopolymerization. The ink system is composed of stoichiometric mixtures of pentaerythritol tetra(3-mercaptopropionate) (PETMP, 4Thiol), triallyl-1,3,5-triazine-2,4,6-trione (TATATO, 3Ene), and an ene-terminated DASA precursor (4-methoxy-N-(pent-4-en-1-yl)aniline, EnePre) from a 2-step synthesis (Figure 1A). Using diphenyl(2,4,6-trimethylbenzoyl)phosphine oxide (TPO) as the photoinitiator, an offstoichiometric thiol-ene photocrosslinking was performed upon exposure to either UV light for one-photon curing or near-infrared laser pulses for 2PP. After color developing these networks in a solution of activated furan adducts (FAs) where the DASAs are formed by reaction with the aromatic amine, photochromic DASA polymer networks were generated (Figure 1A). Three different FA based on Meldrum's acid (Meld), N-substituted barbituric acid (Barb) or a pyrazolone (Pyra) were employed. While the first two FA have been employed already in the first reports of DASA photoswitches,[15] Pyra was the first of a new set of FA, pushing the DASA absorbance towards higher wavelengths.[22] The precursor-based approach offers several advantages: First, it minimizes the risk of photodamage to DASAs during photopolymerization but keeps the capacity to fabricate 3D micro-objects composed of DASAnetworks through nanoscale 3D-printing via 2PP. Second, simple variation of the FA provides access to multiple DASAs with different colors and photoswitching properties from the same precursor network. With the rapid development of new FAs, the approach can be expanded to include novel DASA types with different or improved properties and functionalities. Finally, by varying the stoichiometry of thiol-ene monomers the network structure, composition, and mechanical properties can be tuned to optimize their performance as a photochromic matrix.

To investigate the thiol-ene click photopolymerization and the resulting network properties, we synthesized a number of networks with different compositions by tuning the stoichiometry of the monomers (see Table 1 for samples and their nomenclature). After development of a 
network, for example, 2:1-1 with the Meld towards the Meldrum's acid-based DASAs, it was termed 2:1-1MD. Accordingly, it was termed 2:1-1BD for Barb and 2:1-1PD for Pyra, with MD, BD, and PD designating the Meld-, Barb-, and Pyra-based DASAs (Figure 1B). Due to the intense color of DASAs, with molar extinction coefficients of the open form in the range of $\sim 10^{5} \mathrm{M}^{-1} \cdot \mathrm{cm}^{-1},[10]$ only small amounts of DASAs are typically needed to provide strong coloration.

\section{Network Structure and Mechanical Properties}

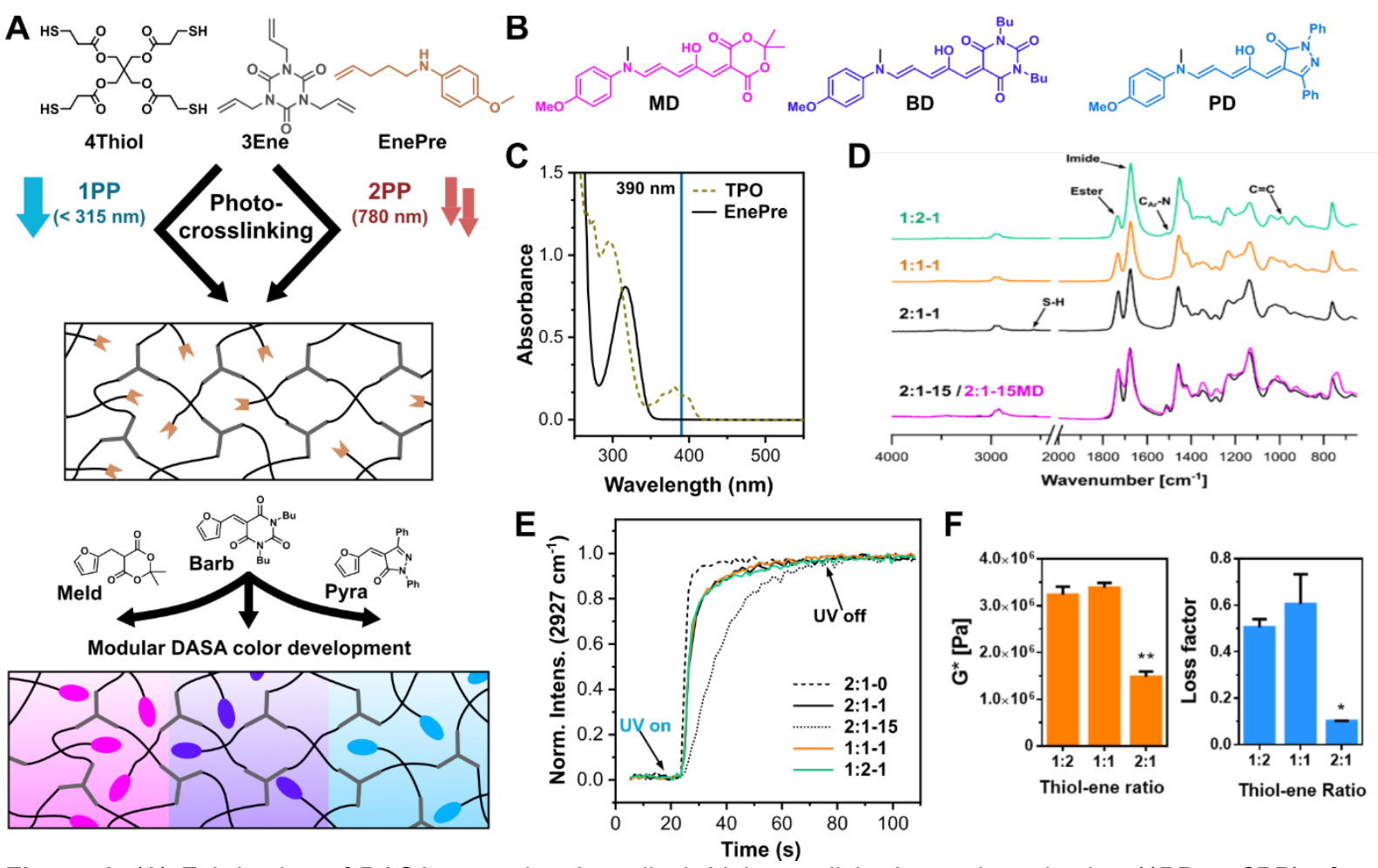

Figure 1. (A) Fabrication of DASA-networks via radical thiol-ene click photopolymerization (1PP or 2PP) of three monomers (4Thiol, 3Ene, and EnePre) for modular color development with three activated furan adducts (FAs): Meld, Barb, or Pyra. (B) Structures of the formed DASAs based on Meld (MD), Barb (BD) and Pyra (PD) FAs, respectively. (C) UV-vis spectra of the TPO initiator and EnePre: single-photon absorption at $390 \mathrm{~nm}$, corresponding to half of the laser center wavelength $(780 \mathrm{~nm})$ for two-photon photolithography is marked with a blue line. (D) ATR-FTIR spectra of precursor networks with different network compositions and one DASA-network. The spectra show key features of the network molecular structures, derived from the precursor monomers (compare with Figure S2). (E) In situ monitoring of thiol-ene photopolymerization kinetics of networks with different compositions using ATR-FTIR. (F) The effect of thiol-ene ratio on matrix mechanics - complex modulus $\left(\mathrm{G}^{*}\right)$ as determined by photo-rheology.

The chemical compositions of our ink system were investigated by attenuated total reflectance Fourier-transformed infrared (ATR-FTIR) spectroscopy. FTIR spectra of the different precursor networks are presented in Figure 1D and the spectra of both monomers and 
EnePre are shown for comparison in Figure S1. The different compositions of the networks are mirrored by the relative intensities of the ester peak of 4 Thiol $\left(1730 \mathrm{~cm}^{-1}\right)$ and the imide peak of 3Ene $\left(1674 \mathrm{~cm}^{-1}\right)$. An excess of ene groups in the monomer mixture (1:2-1) resulted in the complete disappearance of the thiol signal at $2562 \mathrm{~cm}^{-1}$ while an excess of thiol groups (2:1-1) resulted in the disappearance of the ene signal at $924 \mathrm{~cm}^{-1}$. The disappearance of both signals for an equal ratio (1:1-1) indicates a high conversion of the functional groups during thiol-ene photopolymerization. A high degree of monomer incorporation into the network is supported by the small difference between unwashed precursor networks and ones that were washed in organic solvent (Figure S4). The successful incorporation of EnePre was confirmed by the appearance of a characteristic peak of the aromatic amine at $1512 \mathrm{~cm}^{-1}$. Higher concentrations, for example for 2:1-15 and for 2:1-3 were incorporated into the networks as well, demonstrating good control over the degree of network functionalization (Figure 1D, bottom, and SI5).

Next, we investigated the kinetics of thiol-ene photopolymerization (1PP) by in situ ATRFTIR.[38] The peak at $2927 \mathrm{~cm}^{-1}$ corresponds to the formation of a methylene group during the polymerization. The monomer mixture without EnePre reacted extremely fast, being cured after an exposure to UV light for $3 \mathrm{~s}$, indicating the excellent efficiency of radical-mediated thiolene click reaction.(Figure 1E). The presence of EnePre retarded the polymerization in a concentration dependent manner, though it still occurred within few seconds for the practically relevant formulations with 0.01 eq. of EnePre. Notably, the stoichiometry of the monomer components did not have any observable effect on the polymerization kinetics. The retarding effect of EnePre can be explained by the overlap of its absorption spectrum with the one of TPO initiator in the UV region (Figure 1C). However, such an overlap does not exist during the twophoton absorption of TPO at $780 \mathrm{~nm}$. Therefore, 2PP can be expected to occur without interference. Comparison of full spectra from the early and late stages of the polymerization further confirmed that functional groups are consumed during the polymerization process and not removed later during the washing step (Figure S6A). Monitoring at different wavelengths 
corresponding to the $\mathrm{C}_{\text {vinyl- }} \mathrm{H}$ bond $\left(3075 \mathrm{~cm}^{-1}\right)$, the thiol group $\left(2562 \mathrm{~cm}^{-1}\right)$, or the vinyl group $\left(924 \mathrm{~cm}^{-1}\right)$ gave the same result (Figure S6B). Furthermore, independent repetitions of the experiments confirmed excellent reproducibility (Figure S6C).

The physical properties of a polymer matrix, in particular its stiffness/softness and the amount of polymer free volume can impede or allow the required dynamic motion on the molecular level during photoswitching.[39, 40] Therefore, we utilized in situ photorheology (Figure S7) to assess the mechanical properties and photoreactivity of the resulting networks. Soft polymer matrices from low- $T g$ polymers generally provide the best conditions for the photoswitching of photochromic compounds with large changes in molecular shape.[41] Previous reports on DASAs that isomerize from a linear extended form into a cyclic and compact form have emphasized the importance of such matrix properties, with only soft low$T g$ matrices enabling good performances.[15, 25]

The measurements confirmed the fast polymerization kinetics. All changes occurred within $3 \mathrm{~s}$, which is the time resolution of the photorheology setup. The speed of the network formation as determined by photorheology appears to be even faster than in the FTIR experiments. The difference is explained by the use of multi-functional monomers in a step-growth polymerization that results in an early gel point, i.e. network formation when a portion of the functional groups is still reacting, a behavior already observed by us for other multifunctional polymerizations.[42] The mechanical properties of the networks were found to strongly depend on the ratio of the monomer components (Figure 1F, left panel): A much softer network was obtained from the 2:1-1 composition in comparison to the networks from 1:2-1 and 1:1-1 compositions. Both the molecular structure of the monomers and the crosslinking density of the networks determine their mechanical properties. The more rigid structure of 1:1-1 indicates that the crosslinking density plays a major role. Moreover, the more rigid molecular structure of the cyclic 3Ene monomer appears to result in a more rigid network (compare 1:2-1 vs. 2:1-1). With the contributions of both lower crosslinking density and lower amount of rigid monomers, the 
loss factor of 2:1-1 networks (Figure 1F, right panel) is about 0.1 , which is much lower when compared to the other two networks. The lower the loss factor, the weaker the damping properties of the networks.

Furthermore, we employed dynamic rheology to assess the deformability of the networks. Dynamic frequency sweep experiments (Figure S7C) show a clear difference in network structure. For the stiffer networks, the storage modulus ( $\left.\mathrm{G}^{\prime}\right)$ and loss modulus ( $\mathrm{G}^{\prime}$ ') are crosssectioned at high angular frequencies. In contrast, the $G^{\prime}$ of soft networks (2:1-1) remain constantly higher than G' in the whole range, indicating a distinct difference in microstructure compared to the other two networks.

The difference in mechanical properties suggests a difference also in the thermal properties of the networks. To verify this hypothesis, the networks were analyzed by differential scanning calorimetry (DSC). For the softest network 2:1-1, a glass transition temperature $\left(T_{g}\right)$ of $-8{ }^{\circ} \mathrm{C}$ was found, whereas the $\mathrm{T}_{\mathrm{g}}$ of the stiffer networks were closer to room temperature (Figure $\mathrm{S} 8$ ). Interestingly, washing of the 1:1-1 networks resulted in a decrease in $T_{g}$ by several degrees (Table 1). With the loss of a large amount of residual monomer and oligomers highly unlikely, we suggest a decreased ability to cope with the mechanical strain during swelling of this more rigid network with a higher degree of crosslinking density due to the stochiometric ratio of vinyl and thiol functionalities. Accordingly, no such difference was observed for networks 2:1-1 or $1: 2-1$

\section{Photochromic DASA Networks}

The colorless and optically transparent precursor networks were developed into colorful DASA-networks by reaction with FAs. Colorful network structures were obtained as shown with the example of 2:1-1MD in Figure 2A. Monitoring this reaction with 2:1-15 using FTIR revealed that the reaction takes up to several days and no further reaction occurred after $\sim 7 \mathrm{~d}$ (Figure S8). Due to the relatively slow reaction, which is typical for $2^{\text {nd }}$ generation DASAs based on aromatic amines with low nucleophilicity,[22, 25] the degree of DASA 
functionalization can be adjusted not only by the amount of EnePre in the precursor network but also by the duration for the DASA development step. In fact, we limited the reaction time for several samples since fully reacted DASA-networks from a 100:1 ene-EnePre ratio often were already too intense in color to be analyzed by UV-vis spectrometry. Variation of the FA type enabled the generation of three different DASA-networks with peak absorbance values of $562 \mathrm{~nm}(\mathrm{MD}), 590 \mathrm{~nm}(\mathrm{BD})$, and $616 \mathrm{~nm}$ (PD), covering an overall absorbance range of more than $200 \mathrm{~nm}$ (Figure 2B). Importantly, photoswitching occurred for all network compositions and for all DASA types with the complete loss of color under white light irradiation and thermal reversion to the colored state in the dark (Figure S9/10). Figure 2C shows the loss of characteristic absorption peak of DASA in the 2:1-1MD network upon white light irradiation.
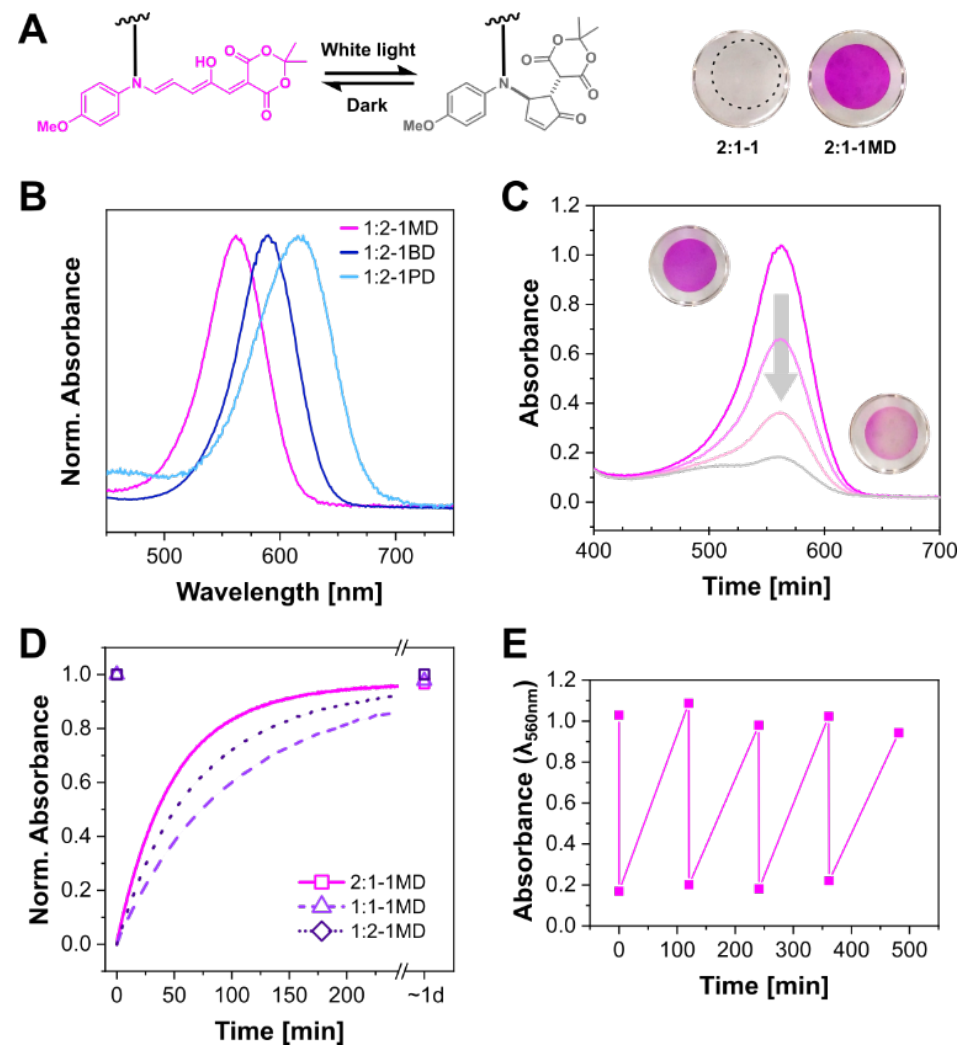

Figure 2. (A) Schematic depiction of the photoisomerization of a DASA-containing network (MD) with open (colorful) and closed (colorless) state. The photos on the right show images of network before and after development (2:1-1 and 2:1-1MD). (B) UV-vis spectra of three different DASA-networks derived from one precursor network. The networks are partially photoswitched to reduce the peak intensity to the range of the spectrometer. (C) Spectra of 2:1-1MD before and after incremental photoswitching by white light irradiation (each spectrum corresponds to additional $10 \mathrm{~s}$ of irradiation). (D) Back-isomerization in the dark of photoswitched DASA-networks of dif-ferent composition. Due to the high absorbance values, the recovery could not be monitored from the peak wavelength for all networks (560 nm for 2:1-1MD, $586 \mathrm{~nm}$ for 1:1-1MD and $520 \mathrm{~nm}$ for 1:2-1MD). Yellow line indicates point of irradiation. (E) Cycling of 2:1-1MD between colorful and colorless state by repeated irradiation (30 s) and recovery in the dark (120 min). 
In our previous work, we found a strong dependency of both the DASA photoswitching and the thermal back-isomerization on the $T_{g}$ of the matrix polymer, with a rigid matrix impeding or even preventing it entirely.[25] As described above, all networks possessed glass transitions below room temperature, and the $T_{g}$ for the $2: 1-1$ network was increased from $-8{ }^{\circ} \mathrm{C}$ to $-5{ }^{\circ} \mathrm{C}$ after the FA reaction (Table 1, Figure S7). To investigate the influence of matrix mechanics on the DASA behavior, we monitored the thermal recovery in the dark for the different network compositions. Interestingly, the recovery rate was found to strongly depend on the specific network type (Figure 2D and Table 1): the $t_{3 / 4}$ (the recovery time of $75 \%$ of the initial absorbance) increased from the soft matrix to $1: 1-1 \mathrm{MD}$ as the most rigid and crosslinked network, suggesting that the soft network favors a fast response. In addition to the network composition, the structure of DASAs also influenced the recovery rate: the Pyra-based PD recovers faster in comparison to $\mathrm{BD}$ and to $\mathrm{MD}$ (Figure S10D). These results are in line with our previous findings $[22,25]$ and underline the importance of tuning the matrix properties as well as carefully choose the DASA type to achieve the desired photoswitching performance.

DASAs are generally susceptible to degradation by strong nucleophiles and, accordingly, free thiols in the networks can result in the degradation of part of the DASA population during the development step or even over time.[12] Indeed, we found an increase in color intensity from the 2:1-1MD network over 1:1-1MD to network 1:2-1MD (Figure S9). The last network has the highest color intensity presumably due to the absence of thiols that may induce DASA degradation. Accordingly we could in the future produce soft networks without residual thiols from a super-stoichiometric amount of thiol. One possibility would be co-macromonomers, for example ene-functional silicones, that increase the softness of the network due to a plasticizing effect.

\section{Nanoscribe 3D-Printing and Dynamic Tuning}

With the superior properties of the 2:1-1 network for photoswitching experiments, we chose this ink for nanoscale 3D-printing of photochromic objects via 2PP. To this end, a commercial 
Nanoscribe GT 3D microprinter was applied to screen the processing window of this ink and to fabricate photochromic objects with user-dictated complex structures (Figure 3A). As shown in Figure 3B, we firstly fabricated simplified line-like structures at varying scanning speeds $(100 \mu \mathrm{m} / \mathrm{s}-10 \mathrm{~mm} / \mathrm{s})$ and laser power $(7.2 \mathrm{~mW}$ to $36 \mathrm{~mW})$. The robust thiol-ene reactions enabled rapid controlled fabrication of reproducible microstructures at writing speeds up to 50 $\mathrm{mm} / \mathrm{s}$, which is the upper limit of the device.

A

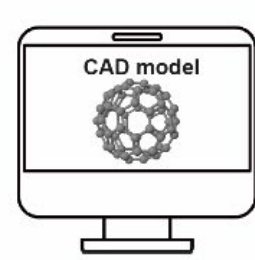

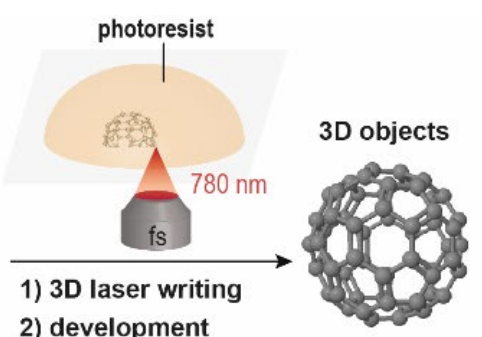

2) development

B

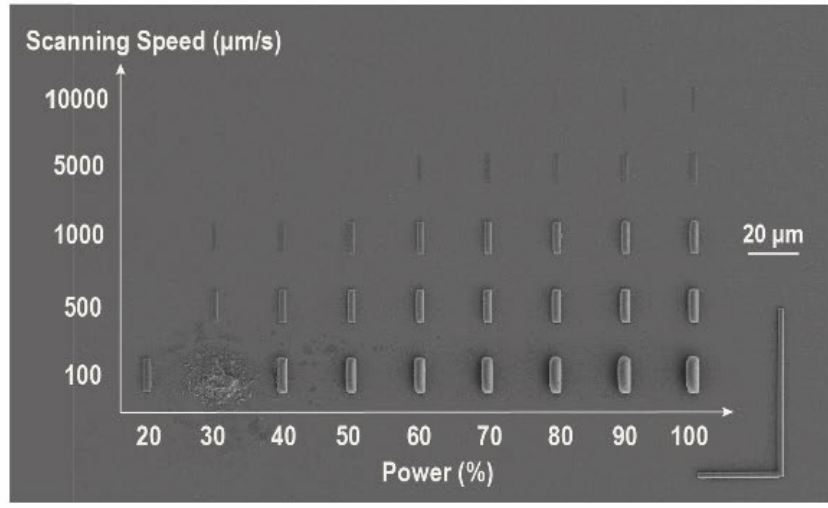

D
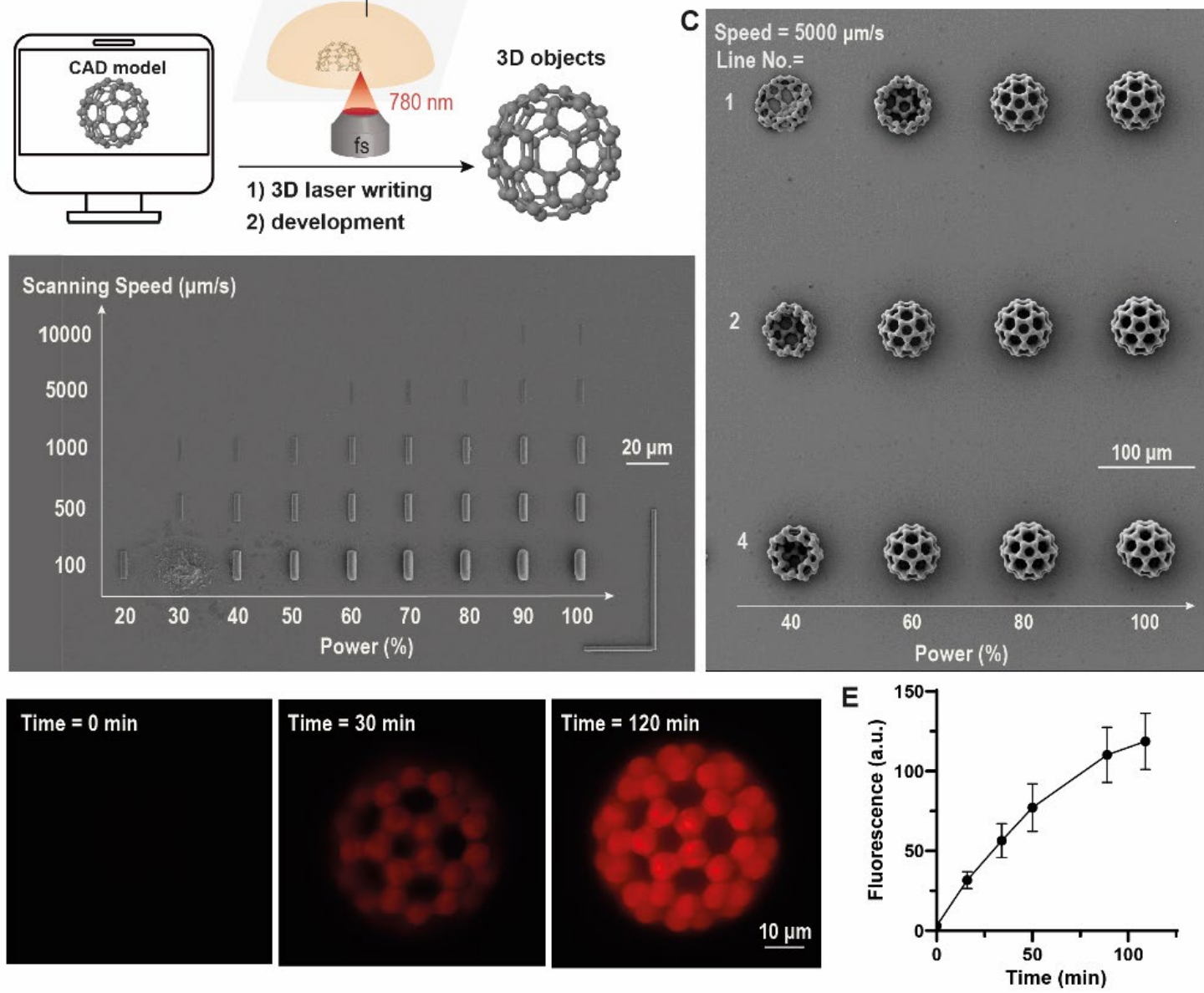

Figure 3. Nanoscribe 3D-printing, functionalization and photo-switching of color-changing fullerene-shaped microstructures. A) Schematic of direct laser writing using a C60-shaped CAD model; B-C) SEM images of microprinted rectangular structures at varying scanning speed and laser power (B) and complex $\mathrm{C} 60$ microstructures at varying line number and laser power (C); D-E) After development, functionalization and laser excitation, the C60 microstructures are photoswitchable. D) Microscopic images of the microstructures at different time points after laser excitation. E) The thermal recovery kinetics of DASA-containing networks could be precisely monitored by the fluorescence.

Furthermore, we attempted to fabricate more complex $3 \mathrm{D}$ micro-objects using a $\mathrm{C}_{60}$-shaped CAD model. Since this model has a spherical shape, the initially printed microstructures tend to collapse or distort, presumably due to the limited interfacial bonding depth $(<1 \mu \mathrm{m})$ despite 
the use of thiol-functionalized glass slide. To address this, we optimized the printing process by increasing the bonding depth to $5 \mu \mathrm{m}$ to enhance the stability of microprinted structures. Furthermore, we found that the increase of LineNumber from 1 to 2 and to 4 could also greatly enhance the structural stability after development in acetone (Figure 3C).

Finally, we studied the feasibility of incorporating DASA into the 3D microprinted networks as well as the photoswitching properties thereof. The printed microstructures were reacted with one type of FA (Meld) for $12 \mathrm{~h}$ under dark to incorporate the DASA into the network. The shortened duration for this reaction is based on the fact the structures are porous structures with sizes of ca. $50 \mu \mathrm{m}$, thereby facilitating the FA reaction. Unlike the samples prepared by 1PP, the printed microstructures have a different appearance. This is likely due to the variations in photoinitiation kinetics and photon intensity between 1PP and 2PP.

Due to the small scale of these structures that renders UV-Vis spectrometry unavailable for their investigation, we decided to employ the fluorescence of DASAs to monitor photoswitching and recovery of nano-3D-printed photochromic structures. Mason et al. have previously shown that the fluorescence of the open form of alkylamine-based DASAs can be used to track the closed-open transition, though this work did not include reversible photoswitching.[21] To our knowledge, no previous work has employed the change in DASA fluorescence to monitor their photoswitching transition in solution or covalently immobilized in a polymeric matrix. Yet it presents a method towards quantitative information on the DASA state even in miniscule intricately and complex structures. First, we investigated the fluorescence of the aromatic amine-based DASA in solution and in 2:1-1MD networks. We found that the DASA solutions and networks have weak fluorescence in the open state (Figure S12), with max. fluorescence of DASA in the open state at $615 \mathrm{~nm}$. Once irradiated with white light, the fluorescent intensity of DASA disappeared due to the transition into the colorless and non-fluorescent closed state upon switching. Furthermore, the recovery of fluorescence in 
DASA networks reached $>95 \%$ of the fluorescence before switching, and $100 \%$ in DASA solutions (Figure S12), much in accordance with our previous UV-Vis experiments.

We applied these findings to characterize the switching behavior of our structures. The 3D microprinted fullerene structures were, first, photobleached by green laser irradiation at $546 \mathrm{~nm}$ for 2 min on a Zeiss LSM880 laser microscope. Subsequently, time-lapse confocal imaging was performed to monitor the fluorescence recovery of DASA-containing networks inside the microstructure. As shown in Fig. 3D, the fluorescence of DASA in the microstructure recovers after $120 \mathrm{~min}$. Moreover, the fluorescence intensity could be quantified and plotted as depicted in Fig. 3E. To the best of our knowledge, this is the first work that demonstrates the fluorescence of DASA photoswitches can be utilized to study the reversible switching and recovery of these photochromes, allowing the reading of DASA-containing polymer matrices in 3D.

\section{Conclusions}

Visible light-responsive polymer networks with covalently attached DASA photoswitches were accessed in two steps via photocrosslinking of a thiol-ene clickable ink into networks and subsequent development of a range of DASAs with different color profiles. Photocrosslinking with UV photoinitiation provided thin films while 2PP with near infrared laser pulses enabled the microfabrication of $3 \mathrm{D}$ objects. The properties of the networks as a photoswitch matrix depended strongly on the stoichiometry of the monomers and the resulting mechanical properties of the polymer networks, with softer networks enabling faster DASA isomerization. The presented methodology provides control over the composition and the geometry of the networks, as well as the degree of functionalization and type of integrated DASAs. Integration of new monomers and comonomers will allow further improvement of the matrix properties in the future to provide optimized photoswitching properties, e.g., by using ene-functionalized silicones. Importantly, the modular nature of the presented approach can be readily expanded with new FAs, or combinations thereof, to design absorbance and photoswitching profiles over the whole visible spectrum. In the future, the combination with fluorescent dyes in the matrix 
can allow both 2-photon-based writing and fluorescence-based reading. With the optimization of the 2PP process on high efficiency commercial microprinters, the presented methodology can enable the writing of photochromic objects for applications as smart multi-addressable optical filters and switches within complex microfabricated structures, with high demands on their exact localization and shape.

\section{Supporting Information}

Supporting Information is available from the Online Library or from the author.

\section{Acknowledgements}

The Swiss National Science Foundation (SNSF) is acknowledged for the financial support through Grant No. 200021_172609. XHQ would like to acknowledge ETH Zurich and Swiss National Science Foundation (SNSF) for the financial support through an ETH Career Seed grant (SEED21 18-2) and a SNSF Spark grant.

\section{References}

[1] Crano, J. C. \& Guglielmetti, R. J. Organic Photochromic and Thermochromic Compounds, vol. 1: Main Photochromic Families of Topics in Applied Chemistry (Kluwer Academic Publishers, New York, 1999), 1 edn.

[2] Russew, M. M. \& Hecht, S. Photoswitches: From molecules to materials. Adv. Mater. 22, 33483360 (2010).

[3] Tian, H. \& Zhang, J. (eds.). Industrial Applications and Perspectives (Wiley-VCH, Weinheim, 2016).

[4] Pauly, A. C. et al. ATRP-based synthesis and characterization of light-responsive coatings for transdermal delivery systems. Sci. Technol. Adv. Mater. 16, 034604 (2015).

[5] Schöller, K. et al. From Membrane to Skin: Aqueous Permeation Control Through Light Responsive Amphiphilic Polymer Co-Networks. Adv. Funct. Mater. 24, 5194-5201 (2014).

[6] Irie, M. Diarylethenes for memories and switches. Chem. Rev. 100, 1685-1716 (2000).

[7] Wang, L. \& Li, Q. Photochromism into nanosystems: towards lighting up the future nanoworld. Chem. Soc. Rev. 47, 1044-1097 (2018).

[8] Xiao, P., Zhang, J., Zhao, J. \& Stenzel, M. H. Light-induced release of molecules from polymers. Prog. Polym. Sci. 74, 1-33 (2017).

[9] Bleger, D. \& Hecht, S. Visible-light-activated molecular switches. Angew. Chem. Int. Ed. Engl. 54, 11338-11349 (2015).

[10] Helmy, S. et al. Photoswitching using visible light: A new class of organic photochromic molecules. J. Am. Chem. Soc. 136, 8169-8172 (2014). 
[11] Helmy, S., Oh, S., Leibfarth, F. A., Hawker, C. J. \& Read de Alaniz, J. Design and synthesis of donor-acceptor stenhouse adducts: A visible light photoswitch derived from furfural. J. Org. Chem. 79, 11316-11329 (2014).

[12] Lerch, M. M., Szymanski, W. \& Feringa, B. L. The (photo)chemistry of stenhouse photoswitches: guiding principles and system design. Chem. Soc. Rev. 47, 1910-1937 (2018).

[13] Yan, Q. et al. Visible light responsive donor-acceptor stenhouse adducts with indoline-tri/tetraphenylethylene chromophore: Synthesis, aggregation-induced emission, photochromism and solvent dependence effect. Dyes Pigm. 178, 108352 (2020).

[14] Hemmer, J. R. et al. Tunable visible and near infrared photoswitches. J. Am. Chem. Soc. 138, 13960-13966 (2016).

[15] Hemmer, J. R. et al. Controlling dark equilibria and enhancing donor-acceptor stenhouse adduct photoswitching properties through carbon acid design. J. Am. Chem. Soc. 140, 10425-10429 (2018).

[16] Mostafavi, S. H. et al. Photoinduced deadhesion of a polymer film using a photochromic donoracceptor stenhouse adduct. Macromolecules 52, 6311-6317 (2019).

[17] Yap, J. E., Mallo, N., Thomas, D. S., Beves, J. E. \& Stenzel, M. H. Comparing photoswitching of acrylate or methacrylate polymers conjugated with donor-acceptor stenhouse adducts. Polym. Chem. 10, 6515-6522 (2019).

[18] Ulrich, S. et al. Electrospun colourimetric sensors for detecting volatile amines. Sens. Actuators, $B$ 322, 128570 (2020).

[19] Chen, Y. et al. Visible light-controlled inversion of pickering emulsions stabilized by functional silica microspheres. Langmuir 34, 2784-2790 (2018).

[20] Jia, S. et al. Investigation of donor-acceptor stenhouse adducts as new visible wavelengthresponsive switching elements for lipid-based liquid crystalline systems. Langmuir 33, 2215-2221 (2017).

[21] Mason, B. et al. A temperature-mapping molecular sensor for polyurethane-based elastomers. Appl. Phys. Lett. 108, 041906 (2016).

[22] Rifaie-Graham, O. et al. Wavelength-selective light-responsive DASA-functionalized polymersome nanoreactors. J. Am. Chem. Soc. 140, 8027-8036 (2018).

[23] Senthilkumar, T. et al. Conjugated polymer nanoparticles with appended photo-responsive units for controlled drug delivery, release, and imaging. Angew. Chem. Int. Ed. Engl. 57, 13114-13119 (2018).

[24] Sinawang, G., Wu, B., Wang, J., Li, S. \& He, Y. Polystyrene based visible light responsive polymer with donor-acceptor stenhouse adduct pendants. Macromol. Chem. Phys. 217, 24092414 (2016).

[25] Ulrich, S. et al. Visible light-responsive dasa-polymer conjugates. ACS Macro Lett. 6, 738-742 (2017).

[26] Wu, B. et al. Visible light triggered aggregation-induced emission switching with a donoracceptor stenhouse adduct. J. Mater. Chem. C 6, 8538-8545 (2018).

[27] Chen, S., Li, W. \& Zhu, W. Multi-addressable Photochromic Materials, 71-108 (Wiley-VCH, Weinheim, 2016).

[28] Lerch, M. M., Hansen, M. J., Velema, W. A., Szymanski, W. \& Feringa, B. L. Orthogonal photoswitching in a multifunctional molecular system. Nat. Commun. 7, 12054 (2016).

[29] Murphy, S. \& Atala, A. 3d bioprinting of tissues and organs. Nat. Biotechnol. 32, 773-785 (2014).

[30] Qin, X.-H., Ovsianikov, A., Stampfl, J. \& Liska, R. Additive manufacturing of photosensitive hydrogels for tissue engineering applications. BioNanoMaterials 15, 49-70 (2014).

[31] Wallin, T., Pikul, J. \& Shepherd, R. 3d printing of soft robotic systems. Nature Reviews Materials 3, 84-100 (2018). 
[32] LaFratta, C., Fourkas, J., Baldacchini, T. \& Farrer, R. Multiphoton fabrication. Angew. Chem. Int. Ed. Engl. 46, 6238-6258 (2007).

[33] Torgersen, J. et al. Hydrogels for two-photon polymerization: A toolbox for mimicking the extracellular matrix. Adv. Funct. Mater. 23, 4542-4554 (2013).

[34] Qin, X.-H., Wang, X., Rottmar, M., Nelson, B. J. \& Maniura-Weber, K. Near-infrared lightsensitive polyvinyl alcohol hydrogel photoresist for spatiotemporal control of cell-instructive $3 \mathrm{~d}$ microenvironments. Adv. Mater. 30, 1705564 (2018).

[35] Hoyle, C. E., Lowe, A. B. \& Bowman, C. N. Thiol-click chemistry: a multifaceted toolbox for small molecule and polymer synthesis. Chem. Soc. Rev. 39, 1355-1387 (2010).

[36] Iha, R. K. et al. Applications of orthogonal "click" chemistries in the synthesis of functional soft materials. Chem. Rev. 109, 5620-5686 (2009).

[37] Kumpfmueller, J. et al. Two-photon-induced thiol-ene polymerization as a fabrication tool for flexible optical waveguides. Des. Monomers Polym. 17, 390-400 (2014).

[38] Qin, X., Wu, Y., Wang, K., Tan, H. \& Nie, J. In-situ synthesis of exfoliated nanocomposites by photopolymerization using a novel montmorillonite-anchored initiator. Appl. Clay Sci. 45, 133138 (2009).

[39] Morimoto, M. et al. Photochromic Bulk Materials (2016).

[40] Shima, K., Mutoh, K., Kobayashi, Y. \& Abe, J. Relationship between activation volume and polymer matrix effects on photochromic performance: Bridging molecular parameter to macroscale effect. J. Phys. Chem. A 119, 1087-1093 (2015).

[41] Evans, R. A. et al. The generic enhancement of photochromic dye switching speeds in a rigid polymer matrix. Nat. Mater. 4, 249-253 (2005).

[42] Boesel, L. F., Reis, R. L. \& San Román, J. Innovative approach for producing injectable, biodegradable materials using chitooligosaccharides and green chemistry. Biomacromolecules 10, 465-470 (2009). 
Table 1. Properties of precursor and DASA-networks.

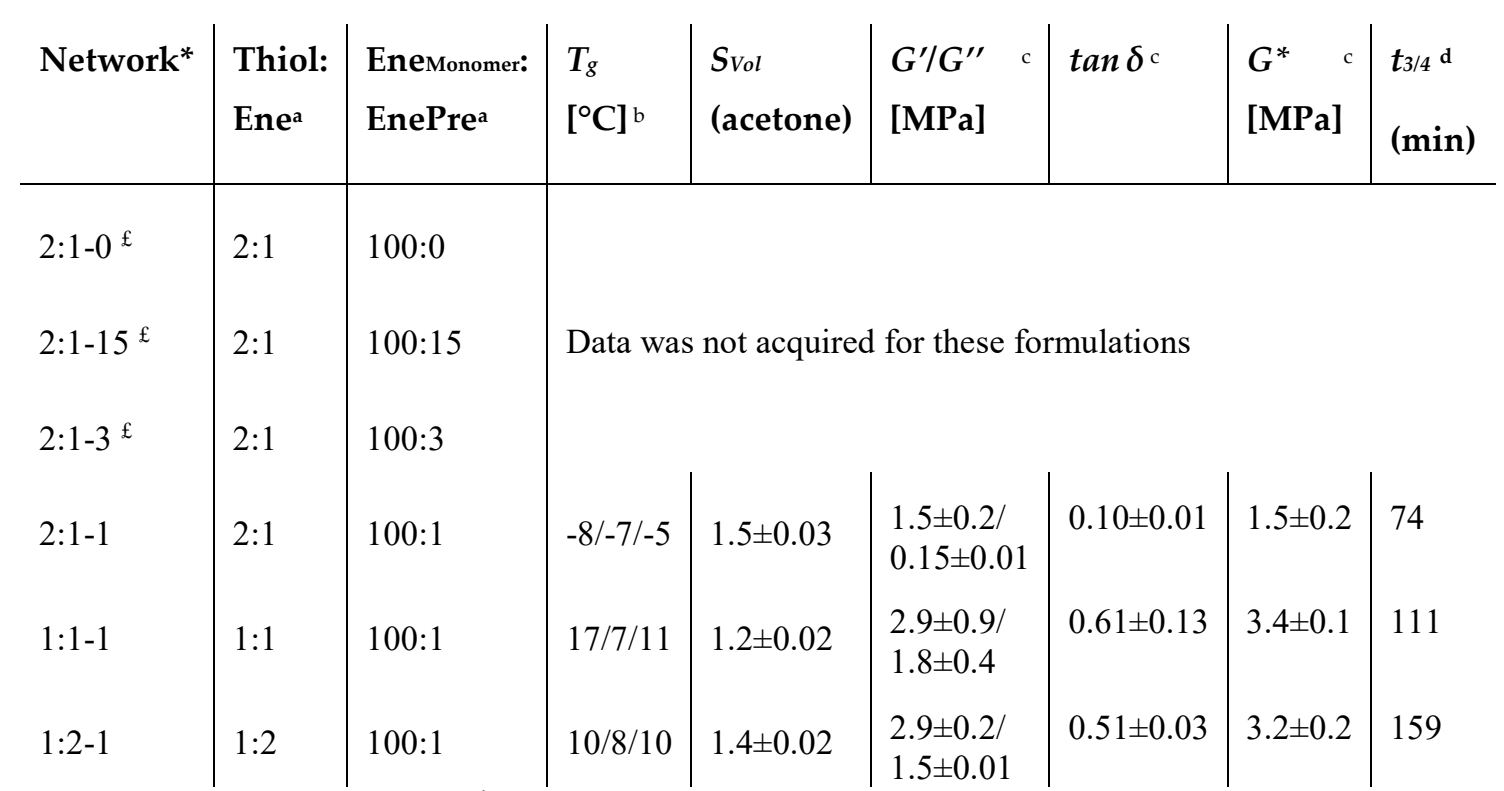

a Ratio in monomer mixture. ${ }^{b}$ Determined by DSC before / after washing of network / after DASA development with Meld. ${ }^{\mathrm{c}}$ Determined by in situ photorheology and dynamic rheology. ${ }^{d}$ Time required to reach $75 \%$ of the final absorbance value during the recovery experiments (Figure 2D).

* The networks are named based on their composition. For example, 2:1-1 refers to a network formed by a monomer mixture with a thiol-to-ene ratio of 2:1, and a 0.01 molar equivalent of EnePre to the ene functional groups of the monomer.

$£$ These networks were used to investigate the influence of particular aspects and are described in the supporting information. E.g., a network with such a high DASA concentration as in the network 2:1-15 had little practical relevance of its own, but was synthesized to increase the visibility of key peaks for the FTIR analysis. 